\title{
Linx
}

Revue des linguistes de l'université Paris X Nanterre

40 | 1999

Le statut d'unité lexicale

\section{Statut lexical, statut lexicographique : le nom composé dans le dictionnaire}

Lexical status, lexicographic status : the compound word in the dictionnary.

Pascale Cheminée

\section{(2) OpenEdition}

Journals

Édition électronique

URL : http://journals.openedition.org/linx/784

DOI : $10.4000 /$ linx.784

ISSN : 2118-9692

Éditeur

Presses universitaires de Paris Nanterre

Édition imprimée

Date de publication : 1 juin 1999

Pagination : 187-217

ISSN : 0246-8743

\section{Référence électronique}

Pascale Cheminée, «Statut lexical, statut lexicographique : le nom composé dans le dictionnaire», Linx [En ligne], 40 | 1999, mis en ligne le 21 juin 2012, consulté le 01 mai 2019. URL : http:// journals.openedition.org/linx/784; DOI : 10.4000/linx.784

Ce document a été généré automatiquement le 1 mai 2019.

Département de Sciences du langage, Université Paris Ouest 


\section{Statut lexical, statut lexicographique : le nom composé dans le dictionnaire}

Lexical status, lexicographic status : the compound word in the dictionnary.

\section{Pascale Cheminée}

1 La linguistique confère un statut sémiotique et sémantique au mot: pour être une unité lexicale, le mot doit permettre de désigner les choses de par sa signification en langue.

01. Le dictionnaire a la - lourde - tâche de rendre compte de l'usage, c'est-à-dire de construire (ou d'inventer, selon le respect ou l'agacement qu'il éveille) le lexique de la langue à partir des discours méticuleusement archivés et organisés dans ses bases de données. Il doit notamment déterminer le degré de lexicalisation de multiples séquences et légiférer. En accordant une entrée - ou une sous-entrée pour bon nombre de syntagmes considérés comme lexicalisés - au mot, il se charge, à travers la définition, de déterminer sa signification de telle manière que l'usager puisse assimiler correctement le lien entre le signe et l'objet du monde par ce nom désigné, c'est-à-dire sa valeur dénominative. Dans le même temps, il se porte garant de la stabilité du signifiant, du signifié et du référent de l'unité ainsi identifiée.

3 Le travail du lexicographe, pour scientifique qu'il puisse être, est voué à l'ambiguïté, pour reprendre le mot d'A. Rey ${ }^{1}$ : tentation (ou vocation ?) de rendre compte du meilleur usage, poids de la tradition, exigences éditoriales et contraintes de toute nature (place, "strates" rédactionnelles, hétérogénéité des rédacteurs...) obscurcissent la description et la rendent inévitablement décevante sur le plan linguistique.

4 02. Les noms composés, difficilement autonomisables sur le plan syntaxique, ont un statut lexicographique mal fixé, que l'on voit varier au gré des éditions. C'est donc un observatoire privilégié pour rendre compte des différences entre statut linguistique et statut lexicographique des unités lexicales. Pour cela, on a choisi d'étudier le nom composé chez-soi auquel le dictionnaire accorde le statut d'unité lexicale puisqu'il l'enregistre en entrée d'article. "Unité » qui, du fait de son instabilité formelle, ne correspond pas aux critères évoqués ci-dessus. On a retenu d'autre part trois syntagmes 
figés, camp(s) de concentration, camp(s) d'extermination, camp(s) de la mort qui, bien que paraissant remplir les conditions requises, ne sont, pour les deux derniers, pas toujours reconnus comme des dénominations, et ont en tout cas un statut lexical instable.

\section{Chez-soi}

1.0. Avec ce mot, peut se poser, bien évidemment, le problème du rapport entre lexique et syntaxe, puisqu'à proprement parler, chez-soi est une séquence syntaxique pétrifiée en quelque sorte par le lexique. On peut se demander, d'un point de vue morphosyntaxique, jusqu'à quel point ce type de transcatégorisés ${ }^{2}$ est devenu un nom. Ce ne sera pas notre angle d'attaque, mais il est intéressant de voir que cette question suscite des interrogations dans d'autres domaines. Nous nous intéresserons aux déterminations sémantiques de ce phénomène : comment la séquence chez-soi, n'ayant pas de stabilité morphologique, peut-elle avoir une homogénéité sémantique? On se demandera jusqu'à quel point ce syntagme remplit les conditions nécessaires pour être une unité lexicale. Il faut croire que cette homogénéité est réelle puisque le syntagme est généralement traité en entrée dans les dictionnaires. Dans ces conditions, on verra sur quoi la lexicographie fait l'impasse par rapport au fonctionnement linguistique de la séquence pour la présenter comme une unité.

\subsection{Chez-soi : quelle « unité »?}

6 1.1.1. "Mieux vaut un petit chez-soi ${ }^{3}$ qu'un grand chez-les-autres »: cet aphorisme populaire, qui joue sur le mot en défaisant ce fragile édifice, et en redonnant à la préposition ses propriétés sémantiques, expose le problème. Il oppose en effet deux syntagmes bien différents: les traits d'union qui relient la deuxième séquence construisent un composé provisoire, ce qui fait ressortir par contraste la différence des deux enchaînements.

7 Le trait d'union qui unit la préposition chez au pronom soi consacre son existence en tant que nom composé, comme signe à valeur dénominative. Mais le jeu de mots, en réactivant les propriétés syntaxiques de la préposition, atteste du même coup que la préposition chez n'a rien perdu de sa valeur sémantique à l'intérieur du nom composé. La même structure syntaxique permet en effet de créer un second composé où les éléments entretiennent les mêmes rapports sémantiques, même si cette nouvelle « unité " n'est pas disponible en permanence dans le lexique.

8 Ici, la maxime joue sur l'instabilité de la forme disjointe du pronom personnel complément, qui tire normalement forme et référence du sujet de la proposition. Elle lui substitue, pour l'y opposer, le pronom indéfini, représentant de la non-identité.

9 Le deuxième élément du composé est donc instable parce qu'il est lié à l'énoncé dans lequel il prend place. Mais il y a un deuxième facteur d'instabilité, plus compromettant pour l'« unité » du nom composé.

10 1.1.2. Il ne manque certes pas de noms composés dont le signifié global peut se déduire du contenu sémantique des éléments en présence. Des sans-abri, des sans-logis sont des $\mathrm{N}$ qui n'ont pas d'abri, pas de logis, pour comparer avec une structure syntaxique analogue.

11 Or ce qui caractérise chez-soi, ce sont d'abord les contraintes d'emploi auxquelles l'unité est soumise. Chez-soi peut se trouver dans une phrase présentative du type : 
1. Voici / c'est mon chez-moi.

dans le contexte $\mathrm{N}+$ adj. :

2. Comme on peut être heureux dans son petit chez-soi !

3. Un chez-soi agréable, accueillant, confortable.

4. La douceur du chez-soi.

Dans les environnements verbaux, chez-soi accepte les constructions directes :

5. Te voilà indépendante maintenant, tu as ton chez-toi.

6. Trouver / chercher un chez-soi.

7. Aimer son chez-soi.

8. Après les vacances, on est bien content de retrouver son chez-soi.

9. Il est des chez-soi qu'on n'a jamais envie de quitter.

En revanche, dans toutes les constructions indirectes où les verbes appellent un syntagme à valeur locative, ce qui ferait se superposer deux prépositions, la construction $\mathrm{V}+$ dét. défini est impossible :

10. * Je reste au chez-moi.

11. * Il va / il rentre au chez-lui.

12. * Je sors / je viens / j'approche du chez-moi.

5 Dans tous ces cas, la seule construction possible qui préserve l'emploi du substantif consiste à utiliser un déterminant possessif précédé de la préposition dans, ou à substituer simplement le déterminant possessif à l'article défini, ce qui produit des énoncés à l'acceptabilité douteuse :

13. ? Je reste dans mon chez-moi.14. ? Il va / il rentre dans son chez-lui.

15. ? Je sors / je viens / j'approche de mon chez-moi.

Les seuls énoncés réellement acceptables sont ceux qui défigent le composé :

16. Je reste chez moi.

17. Il va / il rentre chez lui.

18. Je sors / je viens / j'approche de chez moi.

en lui faisant perdre son statut morphosyntaxique et lexical. La préposition reprend son autonomie chaque fois que le sémantisme du verbe exige une construction qui la capte dans le régime du verbe, comme si cette structure réactivait les propriétés syntaxiques de base de la préposition. Ce serait la même chose pour habiter, conduire, accompagner, recevoir , etc.

17 1.1.3. On voit mal comment une séquence, morphologiquement si dépendante de son environnement, constituerait une " unité ». A priori, les conditions que doit remplir un syntagme pour être une unité lexicale ne sont pas réunies puisque l'association signifiantsignifié / référent se défait, puisqu'elle est notablement instable.

Pourtant, curieusement, cette association remplit l'une des conditions nécessaires pour être reconnue comme nom, c'est la longévité. En effet, la première occurrence habituellement donnée de ce nom composé est celle de 1690, c'est-à-dire la date de son enregistrement dans le dictionnaire de Furetière. Ce mot qui n'en est pas un n'en fait pas moins preuve d'une certaine vitalité ! Voyons donc comment les dictionnaires le traitent.

\subsection{L'entrée}

1.2.1. La forme que prend l'entrée est fonction de la variabilité du deuxième élément du composé. On peut trouver une, deux, trois ou six des variantes morphologiques, soit 
simplement chez-soi (Maxidico), chez-soi, chez-moi ou l'inverse (DFC, Lexis, Hachette) ; chezsoi, chez-moi, chez-toi (PL, PR et RM), ou dans l'ordre grammatical chez-moi, chez-toi, chez-soi pour le GLLF; dans un ordre dicté par la fréquence des occurrences de leur base de données, chez-soi, chez-moi, chez-lui, etc., pour le TLF ; enfin, solution particulière au GLU, d'abord chez-soi, puis dans l'ordre grammatical chez-moi, chez-toi, chez-nous, chez-vous, chezeux: on peut déduire de ce choix, évidemment pas explicité, que le lexicographe considère cette réalisation du composé comme la plus courante, les autres réalisations étant plus aléatoires. C'est aussi le sentiment linguistique qu'on peut en avoir, intuition confirmée par le choix argumenté du TLF. En effet, une remarque en fin d'article du TLF nous informe que "le composé admet tous les pronoms accentués", mais chez-soi représente à lui seul plus de la moitié des 144 occurrences du fond littéraire du TLF, et avec chez-moi et chez-lui, on atteint les trois quarts, ce qui justifie le choix de l'entrée. D'autre part, d'après le TLF, les occurrences ne sont jamais au pluriel.

1.2.2. C'est la présence ou l'absence de trait d'union qui, malgré des exceptions aujourd'hui célèbres telles trait d'union, pomme de terre, chemin de fer et quelques autres, définit les mots composés qui peuvent être des entrées et ceux qui ne sont que des sous-entrées ${ }^{4}$. Si chez-soi n'avait pas de trait d'union chez Furetière, et pas encore dans le Nouveau Larousse Illustré de $1904^{5}$, tous les dictionnaires contemporains lui accordent cet élément graphique qui atteste son unité. Sans doute la " décadence du trait d'union ", selon le mot d'A. Rey ${ }^{6}$, doit expliquer que seules 54 des 144 attestations du TLF en soient porteuses.

1.2.3. Mais le critère du trait d'union ne suffit pas à expliquer le classement de tous les ouvrages. Les Robert font en effet exception : chez-soi n'y est pas traité en adresse mais en sous-adresse avec l'indication de sa catégorie grammaticale. Dans le RM, ce regroupement fait partie du projet lexicographique. Mais quelle est la logique du PR ? On peut supposer qu'elle est historique, puisqu'on le sait, l'histoire guide en permanence l'organisation des articles de cet ouvrage. La préposition chez vient en effet du latin casa, "maison », et le composé signifie donc étymologiquement «la maison de moi, de toi, de lui...». Le Larousse de 1904 n'en donne d'ailleurs pas d'autre définition.

Du DFC et du Lexis, qui inscrivent à leur programme les critères distributionnels, on aurait $\mathrm{pu}$ attendre un regroupement. Ce nom composé étant tellement dépendant de sa structure syntaxique, l'entrée en sous-adresse de chez était justifiée. Or tel n'est pas le cas.

Des noms composés de structure syntaxique équivalente comme sans-abri et sans-logis, lexicalisés respectivement depuis 1935 et 1893, sont traités en entrées dans le PR et en sous-entrées dans le Lexis. Les deux dictionnaires s'accordent à traiter en syntagmes figés à l'entrée soi les concepts philosophiques en-soi et pour-soi, malgré leurs traits d'union, mais ils divergent à nouveau à propos de sur-moi en sous-entrée de moi dans le Lexis, avec un trait d'union, et en un seul mot, surmoi, en entrée dans le PR. Divergence, encore, à propos du non-moi : un concept pour le PR, un avatar du moi pour le Lexis... Ces exemples, qui ne vont pas, on nous l'accordera, sans quelque arbitraire tant à propos du trait d'union qu'en ce qui concerne le "droit d'entrée", montrent en tout cas que la logique qui prévaut au classement n'est pas dépourvue d'opacité.

24 Le composé chez-soi, malgré la variation du deuxième élément, est donc reconnu comme une unité du lexique par l'ensemble des dictionnaires, mais la dissidence des Robert est troublante. 


\subsection{Homogénéité sémantique du nom composé}

\subsubsection{Dans les dictionnaires} longuement développé dans la paraphrase du GLU, semble neutralisé par la mention du lexème domicile à l'entrée demeure, lequel est indexé comme littéraire. Les sémèmes de ces co-définissants étant décrits de façon pratiquement identique, on doit considérer que les dictionnaires en font des unités sémantiquement et référentiellement équivalentes. Pourquoi alors donner deux équivalents s'ils sont synonymes? Le dictionnaire ne s'en explique pas.

Il se trouve que, dans ces trois ouvrages, par la disposition des articles, chez-soi n'a pas de définition autre que celle attribuée à ces deux lexèmes. Si la séquence n'a pas de 
définition, c'est comme si, à proprement parler, il n'y avait pas identification d'un référent à part entière, partant pas de sens lexical, et comme si chez-soi devait se contenter d'un signifié d'emprunt. Or tout lexème isole un référent, le fait d'avoir un nom présuppose l'existence d'une entité extra-linguistique qui lui corresponde, qu'elle soit réelle ou imaginaire.

D'autre part, la synonymie implique une relation réversible : si chez-soi signifie demeure ou domicile, l'inverse devrait être vrai. Or au moins au plan de la représentation lexicographique, cela n'est vrai dans aucun des trois dictionnaires. Chez-soi ne figure pas plus comme définissant que comme synonyme aux entrées demeure et domicile qui bénéficient l'un et l'autre d'une paraphrase définitionnelle. La relation est donc asymétrique entre ces unités.

Bien entendu, on peut invoquer les gains de place que permettent ces équivalences synonymiques dans le dictionnaire. Mais dans tout protocole rédactionnel, ce type de définition est sinon proscrit, du moins déconseillé et doit rester occasionnel. Les unités concernées par de telles descriptions sont donc généralement considérées comme non essentielles, voire marginales, ce qui justifie ce traitement. Ces considérations sont évidemment inacceptables pour un linguiste qui aurait plutôt tendance à envisager ce parti-pris comme un symptôme du fonctionnement de cette unité.

Qu'en est-il des autres modes de description?

\subsubsection{Représentation hiérarchique}

Les autres ouvrages donnent des définitions apparemment paraphrastiques hyperonymiques de chez-soi :

\begin{tabular}{|l|l|l|}
\hline CHEZ-SOI : & (connotation familière) Domicile, lieu où l'on habite \\
\hline & (Hachette) \\
\hline & Domicile, maison où l'on vit (le plus souvent avec une valeur affective) (TLF) \\
\hline & Domicile, maison envisagée dans son intimité (GLLF) \\
\hline \hline DOMICILE : & Lieu où une personne habite (Hachette) \\
\hline & Domicile personnel (avec valeur affective) (PR, RM) \\
\hline & $\begin{array}{l}\text { Lien où une personne habite ou est censée habiter en permanence ou de façon habituelle } \\
\text { (GLLF) }\end{array}$ \\
\hline MAISON : & Lieu ordinaire d'habitation (PR, RM) \\
\hline \hline & \begin{tabular}{l} 
Lieu habituel d'habitation (PL) déf.] Bâtiment ou partie d'un bâtiment où l'on habite ; logement (TLF) \\
\hline
\end{tabular} \\
\hline
\end{tabular}


Ces paraphrases ne définissent cependant pas à proprement parler une classe car les propriétés de la classe ainsi isolée sont très réduites, voire inexistantes. On constate en effet que trois hyperonymes ont été retenus. Il s'agit de domicile, maison, lieu.

Lieu est l'incluant de la définition du dictionnaire Hachette, qui donne d'abord un synonyme avant la définition: domicile. Ici, la définition est parfaitement redondante, puisqu'en comparant la paraphrase de domicile à celle de chez-soi, on s'aperçoit qu'elles sont identiques, à ceci près que le pronom indéfini a été remplacé par " une personne ». On a donc un synonyme redoublé d'une définition hyperonymique qui se trouve être la même que celle du synonyme. La situation est la même que dans le cas des définitions synonymiques précédentes. La seule différence, importante, c'est qu'on a fait précéder la définition d'une remarque d'emploi.

Deux autres paraphrases font apparemment de l'unité une sous-classe référentielle de domicile. Le chez-soi est un « domicile personnel » (PL, PR et RM). L'adjectif « personnel », qui est l'équivalent d'un complément de la forme : "d'une personne », n'indique pas une propriété de l'objet « domicile », mais une relation entre le nom domicile et le référent du mot « personne ». La redondance, là non plus, n'est pas loin, car domicile est par définition le «lieu où une personne habite", et l'on peut se demander ce que serait ce domicile schizophrène d'où la personne serait absente... D'autre part, si « personnel » n'était pas entendu comme un adjectif relationnel, mais avait le sens d'« intime», son association avec domicile, si fortement marqué par son emploi dans la langue administrative ${ }^{8}$, aurait un aspect surréaliste. Là non plus, la définition n'isole pas une classe de référents, et l'on ne peut pas dire que chez-soi soit une "sorte de" domicile. La seule information qui différencie le chez-soi de domicile est, là encore, l'indication du registre (avec valeur affective).

Les paraphrases du TLF et du GLLF emploient l'incluant maison pour définir chez-soi. La deuxième acception de maison, dans tous les dictionnaires, est la maison comme logement. Puisque chez-soi est le "lieu où l'on habite » et maison, le « lieu où l'on loge " (GLLF) ou l'« ensemble des lieux que l'on habite » (Hachette), il est certain que le rapport entre maison et chez-soi n'est pas décrit comme un rapport d'inclusion mais comme un rapport de synonymie. La paraphrase du TLF : « maison où l'on vit » n'est pas redondante, elle indique, parmi les signifiés de maison, celui auquel chez-soi est identifié. Un chez-soi est bien une maison, mais il n'est pas une "sorte de" maison. Il est en effet, comme le dit très bien le GLLF, une «maison envisagée dans son intimité ». C'est le point de vue sur le référent qui change. C'est le nom que l'on donne au référent de maison, de domicile ou de demeure quand on l'envisage « avec une valeur affective». Et dans ce cas, on ne dira pas qu'une « maison envisagée dans son intimité est un chez-soi» mais «s'appelle un chez-soi ». D'ailleurs, il est impossible de remplacer dans un énoncé le nom chez-soi par cette formule définitoire. On peut très bien :

Avoir un chez-soi

La définition ne décrit pas le contenu du signe en termes de référence, mais parle de la langue: le signe chez-soi est le nom que l'on donne à la maison envisagée dans son intimité. Le lexicographe a donc utilisé une définition métalinguistique dans laquelle le signe est mis en rapport avec son signifié et non avec un référent. 


\subsubsection{Connotation} on a :

44 - non marquées :

- Une mise en équivalence synonymique, sans marque d'usage, dans lequel le composé ne peut se distinguer par aucun trait sémique de domicile ou de demeure auquel il est renvoyé. En tant que signe, lui sont attribués le signifié et le référent de ces unités.

- une définition pseudo-logique, sans marque d'usage, qui se confond avec l'incluant domicile.

45

- marquées :

- des définitions logiques identiques à celles de l'hyperonyme, mais accompagnées d'un commentaire métalinguistique qui les en distingue.

- une définition métalinguistique.

Dans le cas des modes définitoires non marqués, l'unité n'a aucune existence dénotative, ce n'est qu'un signe alternatif, pour reprendre les termes de G. Petit (v. infra). Dans le second cas, ce qui différencie chez-soi des trois unités proposées comme équivalentes est uniquement la valeur affective. La question, très proche de celle des noms familiers étudiée par G. Petit ${ }^{10}$, s'en distingue cependant dans la mesure où ce qu'il appelle les «sites énonciatifs » du défini et des définissants sont les mêmes, les mots à connotation affective appartenant souvent, comme ici, au français standard. Ce qui n'empêche pas le dictionnaire, par le biais de cette parenthèse [+affectif], de faire fonctionner l'unité comme si elle appartenait à un dialecte ou un registre de langue qu'il faudrait décoder. Les unités auxquelles chez-soi est renvoyé pour son interprétation ne relèvent d'ailleurs pas du français standard, en tout cas pour domicile, [+ administratif], et demeure, [+ soutenu]. Mais comme elles ne sont pas marquées sur le plan affectif, le dictionnaire les utilise comme un $\mathrm{N}$ non-marqué [...] titulaire "normal" de la relation référentielle, comme il le fait pour les noms familiers. Et l'indication d'un registre de communication a pour effet, exactement comme dans le cas du N FAM, d'[hypothéquer] l'éventuelle autonomie que l'analyse serait disposée à lui consentir ${ }^{11}$.

47 La description qui est faite de chez-soi conduit donc à la situation paradoxale suivante : bien que la fonction dénominative du mot l'impose comme signe-nommant ${ }^{12}$, le traitement 
lexicographique du composé en fait une "unité » privée de statut dénominatif, sans autonomie sémantique ou référentielle, un signe qui n'en est pas un.

Or comme le fait remarquer M.-Fr. Mortureux, ce qui est parfois appelé " connotation » peut apparaître dans la définition comme un trait spécifique ${ }^{13}$. Car chez-soi, si nom marqué il y a, est connu de tout locuteur, lexicalisé : ce [+affectif] n'est pas un effet de sens aléatoire et isolé ${ }^{14}$. Rien n'empêcherait en effet le lexicographe d'intégrer la connotation à la définition en produisant une paraphrase hyperonymique, telle que :

Lieu d'habitation où l'on se sent à l'abri et où se déroule la vie intime.

Mais il ne le fait pas, tout en établissant l'unité, par le biais de l'entrée, dans sa fonction dénominative. Il y a là une sorte de double bind ${ }^{15}$ lexicographique : on reconnaitt l'unité sans la reconnaître.

\subsection{2. Éclairage discursif}

1.3.2.1. Cette attitude du lexicographe fait écho au sentiment des personnes interrogées qui, tout en proposant des définitions logiques, remarquent incidemment : «Ce n'est pas vraiment un nom... c'est utilisé comme un nom... mais c'est un bricolage... »"

Et pourtant, le français ne dispose pas d'autre lexème pour identifier ce concept, concept illustré d'ailleurs par une publicité récente ${ }^{17}$ en deux énoncés :

Envie de chez soi

Envie de Cinna.

Ces énoncés correspondent à deux images : dans la première, une femme arrête un taxi dans une rue parisienne envahie de voitures et grise de pluie; dans la seconde, un chez-soi confortable, aux couleurs chaudes et lumineuses, meublé par Cinna est censé dire ce qu'est un vrai chez-soi.

1.3.2.2. Dans le premier énoncé, chez-soi n'a pas de trait d'union, ni d'article. L'absence du trait d'union, à part l'erreur toujours envisageable, rend impossible à déterminer si le publicitaire a voulu utiliser le nom ou la préposition suivie du pronom. De toute façon, la majorité des occurrences de chez-soi recensées par le TLF ne comportent pas de trait d'union. Mais il semble plutôt que l'instabilité morphologique de chez-soi, à mi-chemin entre lexique et syntaxe, est parfaitement exploitée par la rhétorique publicitaire.

Sans trait d'union, l'interprétation de chez soi peut s'appuyer sur l'ellipse du verbe comme rentrer, auquel cas chez est une préposition. L'image est celle d'une action puisque la femme hèle un taxi pour rentrer chez elle. L'emploi du pronom soi entraînerait alors une reformulation avec un impersonnel : quand la ville se fait si inhospitalière, on (= tout le monde) a envie de rentrer chez soi. C'est alors un énoncé de portée générale. Mais cette interprétation n'inclut pas vraiment la deuxième image. Or c'est une publicité séquentielle.

1.3.2.3. Il s'agit plutôt, comme dans le langage du rêve, d'une condensation : on a envie de rentrer chez soi / on a envie de son chez-soi. L'énoncé autoriserait alors l'une et l'autre interprétations.

Si chez soi est un Nom, l'ellipse porte sur l'adjectif possessif, ellipse obligatoire pour être en parallèle avec le Nom propre, lui, sans déterminant. Au moins en structure de surface, chez soi occupe la place d'un $\mathrm{N}$. Chez-soi devient le premier terme d'une implication logique, et même d'une équivalence dont le second terme est un Nom propre: Si l'on a envie d'un chez-soi, alors on a envie de la marque Cinna; ou encore, qui dit chez-soi dit 
Cinna, et qui dit Cinna dit chez-soi. On dirait en termes linguistiques que chez-soi peut commuter avec Cinna, ce sont des synonymes. Si chez soi n'est pas un Nom, il est difficile de préserver l'interprétation séquentielle de ces deux énoncés.

Chez soi est donc ici comme surdéterminé. Le publicitaire l'utilise comme Nom, tout en exploitant les ressources morphosyntaxiques de la séquence.

Ces images, qui pourraient être un support pédagogique pour enseigner la valeur dénominative de chez-soi à des étrangers, prouvent, s'il en était besoin, que le composé, malgré son instabilité formelle, est bien reconnu par la communauté linguistique comme le nom d'une réalité.

\subsubsection{Sur le plan linguistique}

Cet éclairage discursif est utile pour comprendre que la difficulté d'intégration du composé chez-soi au lexique n'est pas due à sa non-identité sémantique, comme pourrait le laisser croire la consultation des dictionnaires, mais à son origine syntaxique.

60 1.3.3.1. Chez-soi, peut-on penser, est le nom assigné à ce segment de réalité faute de mieux. Or il y a eu tentative d'intégration d'une autre dénomination, au début du XIX ${ }^{\mathrm{e}}$ siècle, pour référer à cette même réalité, et qui s'est trouvée en concurrence avec chez-soi pendant quelque temps. Il s'agit du lexème home, anglicisme introduit dans la langue en même temps que confort et confortable. Si l'on a oublié depuis longtemps l'origine anglaise de ces deux dernières unités, il n'en est rien pour home ${ }^{18}$, qui ne s'est vraiment assimilé que dans le composé home d'enfants. En tant que synonyme quasi absolu de chez-soi, donné par tous les dictionnaires mais signalé comme vieilli, dans l'usage, home est un néologisme qui n'a pas pris et demeure un corps étranger par rapport à la langue française. On peut difficilement invoquer une difficulté d'intégration d'ordre phonétique, puisqu'il existe un homophone, heaume, peu courant il est vrai pour des raisons historiques, mais dont le statut n'est pas problématique. Utilisé mentalement entre guillemets, comme effet de sens, home a un statut dénominatif plus qu'incertain, qui l'apparente au surnom, à l'appellation.

61 De même que l'exemple discursif, ce rappel diachronique montre en tout cas que l'identité sémantique et référentielle de chez-soi est suffisamment assurée pour avoir résisté à la concurrence.

1.3.3.2. Le sentiment de "bricolage» qui subsiste par rapport à ce composé, "pas vraiment un nom », « utilisé comme nom » est donc dû à son origine syntaxique. Or que se passe-t-il dans le cas présent? Le nom composé chez-soi correspond à une phrase du type: c'est un endroit où l'on est chez soi. On pourrait reconstituer une phrase équivalente avec tous les autres pronoms. Il se trouve qu'à un moment donné, la masse parlante est passée de la syntaxe au lexique en estimant que chez-soi constituait le nom d'une catégorie. En créant ce concept grâce à ce nom, la communauté linguistique l'intègre au lexique de la langue. Ce nom a dès lors une existence sociale, collective, il devient disponible à chaque instant, pour tout locuteur, et ce, en dehors de toute énonciation. C'est le parcours de tout néologisme qui s'inscrit au lexique. C'est aussi le parcours de toute séquence d'origine syntaxique qui se lexicalise.

Comme le prouve la violence des réactions à chaque tentative de réforme d'orthographe, et notamment par rapport à l'homogénéisation du pluriel des noms composés, les locuteurs ont du mal à oublier l'origine syntaxique de ces séquences et à les admettre comme des noms ordinaires. Le cas extrême de chez-soi, si peu reconnu comme unité 
lexicale, en dépit de sa cohésion sémantique, en fournit une preuve éclatante. Sa nonintégration notoire permet d'examiner la question comme à la loupe. Mais pourquoi, précisément, chez-soi fait-il, plus encore que la plupart des noms composés, figure de corps étranger dans le lexique?

Normalement, l'opposition centrale entre syntaxe et lexique tient à ce que le contenu d'une phrase n'engage que son énonciateur, c'est-à-dire son auteur et les circonstances dans lesquelles il s'exprime tandis que le lexique implique une reconnaissance collective ${ }^{19}$. La difficulté, en fait, pour tout nom composé, c'est de s'arracher au SN dont il est issu. Bien entendu, pour certains noms composés, comme vinaigre ou gendarme, la lexicalisation est complètement achevée. Mais pour d'autres séquences, bien que reconnues comme unités lexicales, la structure syntaxique est plus présente : sous pèse-lettres, on reconstitue toujours plus ou moins le rapport prédicatif de verbe à complément d'objet, bien qu'on ne soit plus dans une phrase du type : «Je voudrais l'instrument qui sert à peser les lettres » du fait de la lexicalisation du composé. Cet exemple choisi entre mille ne fait que souligner la prégnance de la syntaxe dans le lexique.

1.3.3.3. Ce problème est poussé à son paroxysme avec le composé chez-soi car il se double de la question de la référenciation du pronom. Pour parer à cette difficulté, l'un des dictionnaires lemmatise l'unité en ramenant les formes fléchies du pronom à la forme canonique qui lui sert d'entrée : chez-soi, lemmatisation d'ailleurs à l'œuvre ici même! Cette solution donne à la séquence une certaine légitimité lexicale, et donc lexicographique, mais ne fait que masquer la réalité. Le choix du pronom de la troisième personne, outre le fait que celui-ci correspond à l'usage le plus fréquent, correspond aussi à un désir de neutralisation, puisqu'il s'agit du pronom dit de la non-personne, personne comme on le sait absente de l'instance de l'énonciation. Soi, en composition avec chez, peut apparaître dans des assertions de portée générale, des aphorismes ou des proverbes, tels que la maxime évoquée au début de cet exposé. C'est le seul cas d'emploi autoréférentiel de chez-soi, le seul cas où il fonctionne comme un nom ordinaire. Mais soi est aussi le seul réfléchi, parmi les formes disjointes du pronom, qui renvoie à on. Et dans ce cas, comme la forme disjointe lui, il tire sa référence de l'énoncé où il est inséré, dont il représente anaphoriquement le sujet. Quant aux formes disjointes des pronoms de la première et de la deuxième personne en composition avec chez, elles sont anaphoriques du pronom personnel correspondant, je, tu, nous, vous, qui ne peuvent être identifiés que par référence à l'acte d'énonciation.

1.3.3.4. Chez-soi se trouve donc écartelé entre trois modes de donation du référent, l'un qui vient du lexique, l'autre de la syntaxe et le troisième de l'instance d'énonciation. Chezsoi est en relation quasi osmotique et indifférenciée avec le SN dont il tire sa forme.

\subsection{Bilan}

Pointe extrême du problème des lexèmes composés, chez-soi illustre la difficulté d'intégration de ces unités au lexique de la langue. Malgré son homogénéité sémantique, son instabilité morphologique est un lourd handicap pour prétendre au statut d'unité lexicale. Se fondant dans le $\mathrm{SN}$ dont elle est issue dans les constructions indirectes où le verbe appelle un syntagme à valeur locative, changeant de forme en fonction du sujet de la phrase, la séquence ne retrouve un statut d'unité lexicale à part entière que dans les énoncés où le sujet n'est pas exprimé, comme dans les phrases nominales, les tournures 
infinitives ou impersonnelles par exemple, ou dans les assertions de portée générale qui ont précisément pour caractéristique d'exclure les trois « personnes ».

Pour représenter ce mot qui n'en est pas un, les dictionnaires ont deux solutions : soit ils accordent une entrée à la séquence, ce qui la reconnaît comme unité lexicale au même titre que n'importe quelle autre dénomination. Ce statut pèche par excès, dans la mesure où l'impasse est faite sur la non-autonomie syntaxique de cette « unité ». Soit ils ne lui accordent pas d'entrée autonome, comme le font les Robert, en classant la séquence comme sous-entrée de chez, ce qui rend compte de cette dépendance syntaxique. Mais dans tous les cas, la représentation pèche par défaut, en ce qu'elle ne reconnaît aucune autonomie sémantique et référentielle au composé. Ce faisant, la description lexicographique brouille les cartes en inversant les données du problème.

\section{Camp(s) de concentration, camp(s) d'extermination, camp(s) de la mort}

2.0. Avec les trois locutions camp(s) de concentration, camp(s) d'extermination, camp(s) de la mort, se pose bien sûr la question de l'ambiguïté du statut lexicographique des syntagmes lexicalisés : contrairement à chez-soi, ces trois entités lexicales n'ont pas d'entrée propre, pas plus qu'elles ne sont signalées à leur ordre alphabétique. Elles se trouvent généralement traitées à l'entrée camp. L'impossibilité pratique qu'il y a à multiplier le nombre d'entrées accentue, on le sait, le divorce entre lexème et unité lexicographique.

Mais pour des raisons qui ne relèvent cette fois pas de la syntaxe et qui sont sans doute largement extra-linguistiques, on se trouve ici dans un no mand's land, une zone d'incertitude entre langue et discours, à mi-chemin entre dénomination et désignation ${ }^{20}$. $\mathrm{Si}$, à la différence de chez-soi, ces noms composés constituent des séquences morphologiquement stables, leur statut lexical reste cependant également incertain, et une observation attentive des dictionnaires ne permet pas vraiment de trancher entre lexème composé et syntagme libre.

\subsection{Camp de concentration}

\subsubsection{Rappel lexicographique}

71 Dans l'histoire de la lexicographie française, le syntagme camp de concentration a fait son apparition en $1906^{21}$. L'invention du concept de camp de concentration remonte à la fin du XIX siècle. Pour le Larousse du XXe siècle (1927), les camps de concentration sont des camps de refuge dans lesquels on rassemble, sous la surveillance de troupes, soit des populations civiles de nationalité ennemie, soit des suspects, soit des soldats prisonniers. Si l'on en croit cet ouvrage, les Espagnols en auraient d'abord créé au cours des révoltes cubaines de la fin du XIX ${ }^{\mathrm{e}}$ siècle. Puis, durant la Guerre des Boers (1899-1902), les Anglais parquèrent vieillards, femmes et enfants boers dans des camps de concentration: 25000 d'entre eux moururent par suite de malnutrition et de manque d'hygiène, ce qui souleva alors l'indignation du monde entier.

Toujours selon la même source, les divers belligérants de la Première Guerre mondiale regroupèrent les nationaux ennemis et prisonniers dans de tels camps, mais ceux qui étaient installés en Allemagne donnèrent lieu à de sévères critiques. 
73 Selon le Dictionnaire encyclopédique Quillet (éd. 1988), Le nom de « camps de concentration et de représailles» a été donné par les Allemands à de vastes espaces de baraquements entourés de fortifications et de fils de fer barbelés, flanqués de miradors, où furent enfermés d'abord en 1914-1918 des prisonniers de guerre, puis après 1933, date de l'arrivée au pouvoir de Hitler, des Allemands, ennemis du régime.

74 En croisant ces deux sources, on peut en déduire que les Allemands n'auraient fait qu'aménager et organiser un mode d'incarcération dont ils ne sauraient avoir la paternité. On remarque par ailleurs que l'usage n'a pas retenu et de représailles, le second $\mathrm{S}$ prép en coordination.

75 L'occurrence trouvée dans Proust et citée par le TLF et le GR, extraite du Temps retrouvé22 paru en 1927 :

Vous savez que c'est une affreuse espionne, s'écriait Mme Verdurin [...] Si nous avions un gouvernement plus énergique, tout ça devrait être dans un camp de concentration.

\subsubsection{Analyse du nom composé}

2.1.2.1. Ce balisage historique permet de constater que camp de concentration a été un cas typique de néologie formelle, association inédite d'un signifiant et d'un signifié nouveaux.

L'administration militaire a eu recours, pour ce faire, à l'une des règles de formation habituelle des néologismes, le procédé morphologique de composition nominale. Le nom tête du composé est le morphème lexical camp, dont le sémème relève alors entièrement du domaine militaire. On est avant 1908, et le baron Baden Powell n'a pas encore fondé le scoutisme. Les camps de formation plus ou moins paramilitaires de ses boys n'existent donc pas, pas plus que leur prolongement civil et laïque, le camping, qui fit découvrir à toute une jeunesse entre les deux guerres les bienfaits de la vie en plein air.

78 La valeur dénominative de la base nominale camp sert de noyau pour former camp de concentration. Ceci permet d'intégrer le nouveau signe au paradigme des camps, série lexicale très motivée et apparemment très productive de la terminologie militaire de l'époque, qui avait en effet à sa disposition les synapsies camp de marche, camp de séjour, camp d'instruction, camp de rassemblement, pour ne citer que les unités recensées par la deuxième édition du dictionnaire encyclopédique Larousse ${ }^{23}$.

79 Le composé ainsi formé, dont les deux noms, reliés syntaxiquement par le joncteur " de ", où tous les éléments sont [...] idiomatiques et de forme libre et suivent l'ordre déterminé + déterminant, obéit aux critères de la synapsie décrits par Benveniste ${ }^{24}$. Ce procédé, qui consiste, à spécifier un nom tête, hyperonyme, par le deuxième membre du composé, déterminant ainsi une sorte particulière de camps, est caractérisé par le linguiste comme l'instrument par excellence des nomenclatures, qui limite l'arbitraire du signe en le motivant par rapport à un réseau lexical préexistant: en donnant un nom à ce type de camp, l'administration militaire a rassemblé sous ce signifiant un certain nombre de traits sémiques qui définissent une nouvelle catégorie d'objets. Ce faisant, elle inscrit l'unité dans un réseau d'oppositions distinctives qui enrichit la série lexicale des camps.

80 2.1.2.2. Le statut sémiotique de la séquence ainsi formée est donc clair. Camp de concentration appartient à la catégorie des noms, et comme tel a une signification, une référence virtuelle ${ }^{25}:$ il a la capacité, pour tout locuteur français, d'évoquer un objet de l'univers extralinguistique. Cet objet a certaines propriétés, qu'en tant que lexème, camp 
de concentration condense. Autrement dit, cette unité est caractérisée par sa compositionnalité.

81 La séquence est codée. Son statut dénominatif ${ }^{26}$ se vérifie en ce que l'unité est bien le nom donné à une classe de référents. Elle peut donner lieu à la fois à des questions / assertions métalinguistiques sur le signifié et sur le référent :

\begin{tabular}{|r|r|l|}
\hline & 1) & Que signifie camp de concentration? \\
\hline 2 ) & Quelle sorte de camp est-ce? \\
\hline 3) & Le mot camp de concentration a été créé par l'administration militaire. \\
\hline 4) & $\begin{array}{l}\text { Camp de concentration est le nom d'un endroit où l'on rassemble sous surveillance } \\
\text { militaire des populations civiles, des suspects ou des soldats prisonniers. }\end{array}$ \\
\hline 5) & $\begin{array}{l}\text { Un endroit où l'on rassemble sous surveillance militaire des populations civiles, des } \\
\text { suspects ou des soldats prisonniers s'appelle un camp de concentration. }\end{array}$ \\
\hline
\end{tabular}

Si, pour avoir le statut de lexème, une unité doit être formellement et sémantiquement stable et entretenir une relation dénominative avec un segment de réalité, de telle façon que l'association entre signification et désignation puisse se faire automatiquement, alors la citation de Proust donnée plus haut montre que la séquence remplit ces conditions en tout cas dès 1927.

2.1.2.3. Depuis la Seconde Guerre mondiale, l'usage courant qui est fait du lexème s'applique presque exclusivement aux camps nazis ${ }^{27}$. Son statut lexical a donc changé. Contrairement à l'évolution d'un syntagme comme guerre sainte décrit par S. BrancaRosoff, camp de concentration, sous la pression de l'histoire et dans l'univers de discours qui est le nôtre aujourd'hui, est presque devenu une appellation unique pour un événement particulier $^{28}$, ce qui rend son statut quelque peu hybride, plus proche du fonctionnement d'étiquetage des noms propres que de celui d'un signe à valeur dénominative.

\subsection{Camp d'extermination, camp(s) de la mort}

Depuis la Guerre, les locutions camp d'extermination, camp(s) de la mort sont souvent utilisées comme synonymes de camp de concentration. Mais leur statut lexical actuel, à michemin entre désignation et dénomination, n'est pas clair.

2.2.1. Camp d'extermination est construit comme camp de concentration. La nominalisation peut se gloser de la même manière :

camp où l'on extermine

camp servant à exterminer

Bien entendu, pour un locuteur français d'après-guerre, il s'agit, hélas, d'une association référentielle durable, selon les termes de Kleiber, tous les syntagmes n'ayant pas accédé à la lexicalisation, comme celui-ci, par exemple :

Relégation au ghetto [...] et à partir de 1942, transport dans les camps

d'anéantissement ${ }^{29}$ 

supporter des questions ou des assertions métalinguistiques comme camp de concentration. Le seul test auquel l'unité ne peut répondre de façon satisfaisante, c'est la question 2 :

Quelle sorte de camp est-ce? syntaxique déterminerait une sous-classe de camps, ceux où l'on extermine, ce qui l'intégrerait au paradigme des camps, parmi les camps de marche, de séjour, d'instruction, pour ce qui est des camps à caractère militaire, mais aussi, et avec un humour noir mais sans anachronisme, à celui des camps de vacances et autres camps scouts. S'il existe une relative isotopie sémantique entre les camps de formation militaire et les camps de loisirs, dans le cas d'extermination, il y a dysharmonie totale entre les champs notionnels en cause. On ignore l'origine de ce syntagme dont la première occurrence serait de 1946, mais si c'est une création d'opposants au régime nazi, c'est un procédé qui relève de l'effet discursif et de la qualification : le nom camps de concentration - par définition un lieu où l'on rassemble des prisonniers pour les surveiller - est dans le discours nazi un euphémisme. Dans les faits, il y a eu très souvent une quasi identité référentielle entre les deux noms composés, et le nom camp de concentration signifiait dans bien des cas camp d'extermination. Les camps de concentration nazis n'appartiennent pas plus au paradigme des camps que les camps d'extermination. Utiliser le même pattern syntaxique que pour camp de concentration revient à dénoncer la distorsion perverse introduite par les nazis entre signification et référence.

2.2.2. Beaucoup plus fortement que camp de concentration dont il n'a pas le passé de signe à valeur dénominative, camp d'extermination a un statut proche du nom propre et de son fonctionnement d'étiquetage. Dans l'usage, il n'a aucune valeur généralisante et réfère exclusivement aux camps nazis, de la même façon qu'on utilise le mot goulag pour parler des camps russes.

91 Le syntagme camps de la mort a plusieurs particularités dont celle-ci : il se trouve presque toujours au pluriel, ainsi que l'abréviation équivalente par laquelle il est très souvent reformulé, comme dans cette légende de photographie ${ }^{30}$ :

Vers les camps de la mort. ...ces petits et leurs mères descendent de leur prison roulante pour entrer dans les camps, dont la plupart [...] ne sont jamais revenus.

Le pluriel efface la particularité pour insister sur la généralité du phénomène. L'emploi de l'article défini, qui présuppose une référence unique et connue de tous, ne fait que souligner le poids d'un destin collectif, anonyme.

Le syntagme est a priori une métaphore poétique, frappante par son absurdité. Contrairement aux deux autres noms composés, il y a un article devant le déterminant, ce qui inviterait à interpréter la séquence comme un complément de nom, indiquant l'appartenance. Comme si la mort était une personne qui posséderait les camps. Ce qui peut se paraphraser par:

camps qui appartiennent à la mort

camps où la mort règne

camps où l'on a rendez-vous avec la mort 
94 L'unité peut difficilement répondre à la question 6 ni entrer dans un énoncé de type
définitoire $(7 / 8)$ :

95 Les énoncés 7 et 8 , réponses à la question 6 , sont acceptables, mais ce sont des prédications d'identité et non des prédications d'attribution. Les seules questions / assertions que l'unité peut supporter sont celles où le mot est en usage autonymique, celles qui mettent le signe camps de la mort en rapport avec son signifié :

\begin{tabular}{|l|l|l|}
\hline & 9) & Que signifie camps de la mort? \\
\hline & 10) & Le mot camps de la mort désigne les camps de concentration nazis. \\
\hline & 11) & Camps de la mort est le nom donné aux camps de concentration nazis. \\
\hline
\end{tabular}

Les énoncés 10 et 11 mettent en évidence la différence de comportement entre les deux noms composés. Camp de concentration, on l'a vu, est référentiellement autonome. Il détermine une sous-classe de camps, et à ce titre relève d'une définition logique, même si l'histoire l'a presque vidé de sa fonction généralisante.

2.2.3. Tout se passe comme si l'unité camp de concentration pouvait seule prendre en charge ce statut de nom («name»), alors que pour camps de la mort, comme d'ailleurs pour camp d'extermination, la relation référentielle ne s'établirait que dans un univers de discours précis, celui de la dernière guerre.

On ne peut parler à ce propos de désignation puisqu'il s'agit bien d'une habitude associative récurrente, partagée par tous les locuteurs français, et donc d'un ensemble lexical codé. Ce qui nous dispense, n'en déplaise à certains, d'asserter l'existence du référent ${ }^{31}$ des camps d'extermination / de la mort. Mais on ne peut pas parler non plus de dénomination parce que ces composés ne tirent leur référence que d'un cadre énonciatif unique.

L'interprétation de ces deux séquences camps d'extermination / de la mort ne peut se faire qu'en référence aux camps de concentration qui se trouve, par rapport à elles, en position de nom de signifié pour employer l'expression de J. Rey-Debove. C'est pourquoi il y a inégalité de statut entre les séquences, les dernières ne possédant, en fait d'intension, que celle de la synapsie camp de concentration, et partageant avec les Noms propres le fait d'être attachées à un phénomène unique. 


\subsection{Traitement lexicographique}

100 2.3.0. Le traitement lexicographique des trois syntagmes exhibe ${ }^{32}$ d'une certaine façon, par la diversité des solutions qui sont apportées, la question qui vient d'être évoquée et donne la possibilité en effet d'observer le statut instable des entités lexicales ${ }^{33}$. Ces trois mots composés trace[nt] les limites dans la langue (et / ou dans le discours) entre mot et non mot, et notamment, ici, ne permettent pas de caractériser le mot composé par rapport au syntagme libre $e^{34}$, ou, en d'autres termes, de trancher entre désignation et dénomination. Quel est le degré de codage et d'autonomie de ces unités ? Les neuf dictionnaires ${ }^{35}$ observés donnent presque autant de réponses différentes. Si l'on y cherche un oracle, c'est celui de la Pythie ${ }^{36} \ldots$

101 Étant donné la place qui nous est impartie, nous nous efforcerons de résumer les résultats de la recherche. Concernant ces syntagmes, on est obligé de prendre en compte l'évolution de la description lexicographique.

\subsubsection{Enregistrement des locutions}

Il est difficile de ne pas faire, en commençant, une remarque d'ordre sociolinguistique. Il est très frappant de constater, si l'on prend le dictionnaire comme un texte témoin et reflet de la culture dans laquelle il éclot, combien ce qui est dit, la réflexion menée, le « discours » concernant les camps - et qui transparaît dans les articles - a changé en vingt ans. Peut-être parce que, le temps passant, il devient possible de mettre des mots sur l'innommable. En tout cas, l'exceptionnelle lenteur du dictionnaire à enregistrer «les mots pour le dire ", son silence sont probablement révélateurs de la difficulté à penser ces réalités.

103 Camp de concentration est défini sans aucune référence à la Guerre dans le DFC (1966), dans le Lexis (1971), dans le GLLF (1971). La première allusion se trouve dans l'exemple du GLLF où apparaît l'adjectif nazis. Le syntagme est rapporté à la Guerre dans les Robert, en tout cas à partir de 1985, et il faut attendre les années 90 pour le PL.

Deux dictionnaires sur neuf (GLLF, Hachette 1980-98) ignorent camp d'extermination, absent des deux entrées du composé, le DFC et le Lexis le traitent comme une simple collocation, et cinq sur neuf n'enregistrent pas camp(s) de la mort (DFC, Lexis, GLLF, PL 98, Hachette), comme s'ils ne pouvaient se porter garants du degré de lexicalisation de ces syntagmes. Ils leur refusent de ce fait toute légitimation, toute valeur dénominative.

\subsubsection{Flottement graphique : pluriel}

En principe, si le pluriel est seulement l'addition à la définition sémantique du mot du composant de "pluralité», les dictionnaires n'enregistrent pas une entrée ou une sous-entrée distincte au pluriel. C'est seulement s'il apparaît comme un terme spécifique comportant d'autres traits sémantiques ${ }^{37}$. Or il y a divergence dans les ouvrages à ce sujet.

Camp de concentration est au singulier partout sauf dans le TLF, où il figure en sous-entrée pluriel de l'article concentration (et ce, malgré un exemple de Green au singulier).

107 Camp d'extermination et camp de la mort sont enregistrés au singulier dans le GLU et le TLF mais au pluriel dans les Robert. Par ailleurs, le RM et le PR sont les seuls ouvrages qui enregistrent, à l'entrée camp, après le traitement des locutions camps d'extermination et 
camps de la mort, un emploi « absolu» de camps: Absolt « les camps». Ici, le dictionnaire légitime, reconnaît l'existence d'une unité pluriel camps, sans expansion nominale ou adjectivale, qui est le nom en langue permettant de référer à cette réalité particulière, et qui est en relation d'hyperonymie avec les locutions camps d'extermination et camps de la mort, qu'elle peut reprendre anaphoriquement : le pluriel fait du signe camps un terme spécifique, muni de traits sémantiques particuliers.

Néanmoins, la dysharmonie de traitements prouve qu'il n'y a pas de certitude lexicographique sur l'appartenance du trait pluriel au signifié des unités.

\subsubsection{Statut dénominatif des trois syntagmes dans le dictionnaire}

2.3.3.1. Camp de concentration est défini par tous les dictionnaires majoritairement au second composant, parfois aux deux entrées. Toutes les définitions sont de type hyperonymique et consistent souvent en une analyse de la synapsie : « camp où... », ce qui intègre camp de concentration au paradigme des camps (Hachette, article concentration du PL, du GLU et du TLF). Les allusions à la Seconde Guerre, quand il y en a, sont localisées dans les exemples. Quel que soit le définissant choisi, la locution est décrite comme un signe à valeur dénominative, référentiellement autonome.

Cependant, les PL 91 et 98 font exception : dans l'article concentration, la rubrique HIST. (vague d'ailleurs puisque sans référence à un contexte historique précis ${ }^{38}$ ) qui précède la définition du nom composé oblitère son statut dénominatif. Elle est l'équivalent d'une définition de type métalinguistique "nom donné à», ce qui la met du côté des désignations. Pour être une dénomination inscrite en langue, une unité lexicale ne peut être référencée à un univers de discours particulier.

111 Le TLF fait deux définitions, l'une hyperonymique, «sortale », qui autonomise le lexème, l'autre métalinguistique: "synonyme de camps d'extermination», précédée d'une parenthèse [sous l'Allemagne nazie], qui rapporte le nom composé à une énonciation particulière.

112 Mais l'enregistrement au pluriel est comme une trace de la contamination du signifié par l'histoire. C'est comme un lapsus du lexicographe, qui, tout en s'efforçant de distinguer deux signifiés, ne peut s'empêcher de penser qu'il n'y en a plus qu'un. Le pluriel, en tout cas apporte un flou sur cette distinction et semble semer le doute sur l'existence d'une réelle valeur dénominative de l'unité en dehors d'une référence à la Guerre.

113 2.3.3.2. Camp d'extermination n'est pas défini par cinq dictionnaires sur neuf. En ne l'enregistrant pas (GLLF, Hachette), ou en le traitant comme une simple collocation (DFC, Lexis), ces ouvrages semblent ne pas pouvoir se porter garant de son degré de lexicalisation, ni pouvoir trancher entre syntagme libre et nom composé. Ils ne lui accordent pas de valeur dénominative.

114 Des quatre ouvrages qui définissent le syntagme, seuls les Robert lui accordent droit de cité en le décrivant comme un lexème. La définition, morphosémantique ${ }^{39}$, fait du syntagme un hyponyme de camp de concentration.

La définition du GLU (qui est aussi celle de camp de la mort) : "noms donnés à certains camps de concentration pendant la Seconde Guerre mondiale », de type métalinguistique, prouve que le lexicographe ne considère pas ces séquences comme des dénominations au même titre que camp de concentration. Au niveau du concept et de la chose nommée, les 
signes ont la même dénotation, mais c'est le point de vue sur le référent qui change et c'est lui qui est à définir. Mais le sens est porté par camp de concentration.

Le traitement du TLF est ambivalent: à l'entrée camp, camp d'extermination est traité comme une dénomination. La définition est constituée d'une paraphrase synonymique, échangeable contre la locution, ce qui lui confère un statut lexical de plein droit. Par contre, le traitement de la séquence à l'entrée extermination en fait, sinon un syntagme libre, du moins une habitude associative non codée : ce qui est considéré comme codé est le deuxième composant du mot, à savoir d'extermination. Le premier composant est libre car il peut commuter dans cet environnement par exemple avec guerre. Ici, donc, le syntagme a et n'a pas de statut lexical, c'est un lexème et ce n'est pas un lexème. Linguistiquement, en tout cas, le dictionnaire ne permet pas de trancher si on a affaire à un lexème composé ou à un syntagme libre. En d'autres termes, on hésite entre désignation et dénomination.

117 2.3.3.3. Le TLF est le seul ouvrage à accorder un statut dénominatif au syntagme camp de la mort, défini comme un "camp d'internement où les Allemands exterminaient les ennemis du régime nazi ». Le GLU lui donne la même définition métalinguistique que camp d'extermination. Quant aux Robert, ils distinguent clairement deux lexèmes composés, justiciables d'une définition paraphrastique, l'un, camp de concentration, concernant les "temps de guerre ou de troubles", l'autre, camps d'extermination, la Seconde Guerre mondiale. Camps de la mort n'a pas d'autonomie propre puisqu'il se confond avec camp d'extermination par lequel il est défini.

\subsection{Bilan}

118 On voit donc, d'une édition à l'autre, ces trois syntagmes changer de statut ${ }^{40}$. Bien que n'ayant pas d'entrée autonome, le nom composé camp de concentration voit son statut dénominatif légitimé à la fois par le type de présentation matérielle (sous-adresse en petites capitales que pratiquait Furetière ${ }^{41}$ dans le PR ; acception numérotée - DFC, Lexis, PL et TLF -) mais aussi par les définitions paraphrastiques dont il bénéficie. Pour l'instant, le statut lexical de camp(s) d'extermination et camp(s) de la mort dans les dictionnaires est moins assuré, l'on se trouve à l'interface de la langue et du discours, et, entre lexème composé et syntagme libre, chaque dictionnaire tranche à sa manière en opérant son classement. Si, pour l'instant, le statut de camp(s) de la mort semble n'établir de relation référentielle qu'à l'intérieur d'un cadre énonciatif donné, la situation semble plus contrastée pour camp d'extermination, d'autant que le plus récent des dictionnaires observés (PL 1998), dans son article camp, enregistre un néologisme sémantique qu'aucun locuteur français n'ignore aujourd'hui ${ }^{42}$. Le mot camp s'enrichissant d'un nouveau sémème, ces locutions camp de travail, de concentration, d'extermination deviennent cohyponyme de camp. Dans ce cas, la reprise anaphorique désormais possible de toutes ces locutions par le lexème et hyperonyme camp atteste le caractère lexical de ces syntagmes, en stabilise le statut dénominatif.

\section{Conclusion}

119 Envisagés sous l'angle du statut lexical, le mot composé ou la locution ont des statuts linguistiques et lexicographiques qui ne se recouvrent donc pas, que ce soit pour des raisons formelles, sémantiques ou idéologiques. 
L'analyse linguistique, au plan de la fonction lexicale, a isolé deux types de syntagmes, l'un ayant les deux valeurs du mot, qui lui permet de signifier et de désigner, ce qui en fait une unité lexicale à part entière, les autres ayant un mode de référenciation voisin de celui du Nom propre, plus étiquettes que noms.

Du côté lexicographique, si l'on ne peut ignorer la lourde charge affective et idéologique qui pèse sur ce type de syntagmes et qui peut être partiellement responsable de la hâte ou du retard à l'enregistrement, aussi bien que de la disparité de traitement, on constate néanmoins que les statuts sont variables. Ne leur accordant pas d'entrée, les enregistrant tantôt au singulier, tantôt au pluriel, le dictionnaire les traite soit en lexèmes composés, soit en simples collocations, en désignations, ou en les oubliant de la nomenclature, ne se porte pas garant de leur lexicalisation.

Si des motifs idéologiques peuvent donc être des facteurs de brouillage pour la description lexicographique, des raisons d'ordre linguistique rendent quasi incompatibles statut lexical et statut lexicographique. Le dictionnaire actuel, avec son mode de représentation binaire - une entrée / une définition -, ce qui a pour effet d'autonomiser une unité, peut difficilement rendre compte des séquences qui, si elles sont sémantiquement autonomes, ne le sont pas syntaxiquement, et restent partie prenante et du lexique et de la syntaxe. En fait, tout ce qui est polylexical, à l'exception de quelques célèbres locutions comme pomme de terre ou chemin de fer aujourd'hui en entrées, se voit presque toujours renvoyé en sous-entrée.

À travers l'observation des statuts lexical et lexicographique de ces noms composés, entre ce que le linguiste et le lexicographe appellent un nom, on serait tenté de penser qu'il y a homonymie de concept.

\section{Dictionnaires}

\section{BIBLIOGRAPHIE}

BENVENISTE Émile (1974) Problèmes de linguistique générale, éd. Gallimard, coll. « Tel », Paris.

BONAFOUS S. (1998) Des mots en liberté, E.N.S. éd. Fontenay / St-Cloud, in Mélanges Maurice Tournier, t. I, pp. 39-47.

BOSREDON B. (1991) Prédicativité et lexicalisation, U.A.M. Poznan, in Studia Romanica Posnaniensia , vol. 16, pp. 85-100.

BRANCA-ROSOFF Sonia (1998), Le mot comme notion hétérogène, P.U.P Aix-en-Provence, Langues et langage $\mathrm{n}^{\circ} 7$, in Le mot : analyse du discours et sciences sociales, pp. 7-39.

CHEMINÉE P. (1996) Désignation et signification : paradigmes désignationnels et réseaux analogiques, Thèse de doctorat sous la direction de M. Fr. Mortureux, Paris X Nanterre.

DUBOIS J et Cl. (1971), Introduction à la lexicographie, éd. Larousse, coll. « Langue et langage ", Paris. 
KLEIBER G. (1984), Dénomination et relations dénominatives, Larousse, in La dénomination, Langages $n^{\circ} 76$, pp. 77-94.

MAZIÈRE F. (1996), Le mot au risque du dictionnaire. Sommes-nous tous des étrangers de la langue maternelle ?, CRL Université de Paris X Nanterre, Linx, $\mathrm{n}^{\circ} 8$ spécial, in Du dire et $d u$ discours, Hommage à Denise Maldidier, pp. 13-23.

MILNER J. Cl. (1976), Réflexions sur la référence, Larousse, Langue française $n^{\circ} 30$, in Lexique et grammaire, pp. 63-73.

MORTUREUX M.-Fr. (1997), La Lexicologie entre langue et discours, éd. Sedes, coll. « Campus », Paris. PETIT Gérard (1998), Approche lexicale et sémantique du vocabulaire familier, éd. Didier-INaLF, in Cahiers de lexicologie.

REY Alain (1977), Le Lexique : images et modèles, Armand Colin, Paris.

REY Alain (1983), La lexicographie : rétrospective et perspectives, P.U.L., Lexique 2, in Le Dictionnaire, pp. 11-24.

REY-DEBOVE J. (1971), Étude linguistique et sémiotique des dictionnaires français contemporains, éd. Mouton, Paris.

Dictionnaire alphabétique et analogique de la langue française, Paris, éd. Le Robert, 1985.

Dictionnaire du français contemporain, Paris, Larousse, éd. 1966.

Dictionnaire Hachette encyclopédique, Paris, Hachette, éd. 1998.

Dictionnaire historique de la langue française, Paris, éd. Le Robert, 1992.

Grand Larousse de la langue française, Paris, Larousse, 1972.

Grand Larousse Universel, éd. 1993.

Le Lexis, Paris, Larousse, éd. 1987.

Le Maxidico, Paris, Éditions de la Connaissance, éd. 1998.

Le Petit Larousse illustré, éd. 1991, 1994, 1998.

Le Petit Robert, Paris, Le Robert, éd. 1994.

Le Robert méthodique, Paris, Le Robert, éd. 1986.

Nouveau Larousse illustré, 7 vol., Paris, Larousse, 1897-1904.

Le Trésor de la langue française, éd. du CNRS.

\section{NOTES}

1. Rey Alain, «La lexicographie française : rétrospective et perspectives », P.U.L., Lexique 2, in Le Dictionnaire, p. 13.

2. Ceci est le terme qu'emploie Françoise Kerleroux dans son ouvrage La Coupure invisible: études de morphologie et de syntaxe (Villeneuve-d'Asq, Presses Universitaires du Septentrion, coll. "Sens et structure ", 1996) où elle étudie d'un point de vue morphosyntaxique des enchaînements d'unités lexicales comme rendez-vous, décrochez-moi-ça, etc.

3. Souligné par nous.

4. Dubois J et Cl., Introduction à la lexicographie, Paris, éd. Larousse, coll. «Langue et langage », 1971, p. 62. 
5. Le Nouveau Larousse Illustré traite l'unité en « locution substantive », sous-entrée de chez.

6. Rey Alain, Le Lexique : images et modèles, Paris, Armand Colin, 1977, p. 22.

7. Rey-Debove J., Étude linguistique et sémiotique des dictionnaires français contemporains, Paris, éd. Mouton, 1971, p. 232.

8. Pour une analyse détaillée, voir Cheminée P., Désignation et signification: paradigmes désignationnels et réseaux analogiques, Paris X Nanterre, Thèse de doctorat sous la direction de $\mathrm{M}$. Fr. Mortureux, 1996, pp. 309-316.

9. On prend ici connotation au sens où l'entend le Dictionnaire de linguistique (Jean Dubois et alii, Paris, Larousse, éd. 1989, p. 115) : Référence est faite au contenu émotionnel du vocabulaire pour définir la connotation. Pendant que l'événement culturel symbolisé par le mot fournit la dénotation, le contenu émotionnel du mot, ressenti dans une culture donnée et forgé par cette culture, est à la base du sens connotatif. L'existence même du sujet parlant implique que tout mot possède un contenu connotatif.

10. Petit Gérard, "Approche lexicale et sémantique du vocabulaire familier", in Cahiers de lexicologie, éd. Didier-INaLF, Paris, 1998.

11. Petit Gérard, op. cit.

12. Rey-Debove Josette, op. cit., p. 232.

13. Mortureux M.-Fr., La Lexicologie entre langue et discours, Paris, éd. Sedes, coll. « Campus », 1997, p. 94 .

14. Les traits pertinents de définitions naturelles fabriquées par des locuteurs interrogés pour la circonstance prouvent assez que certains d'entre eux sont reconnus par la communauté linguistique comme relevant de la dénotation : Un chez-soi, un chez-toi, c'est là où tu fais ta vie, c'est ta maison. / C'est un intérieur douillet, personnalisé, tranquille. / C'est le lieu de l'intimité. / C'est une maison qu'on possède, où l'on est protégé, en sécurité. / C'est un lieu stable, un espace, un logis isolé de la société, comme une carapace, une protection.

15. ou « double contrainte » : Situation dans laquelle un partenaire émet simultanément deux messages contradictoires. Ce concept sert à définir le réseau anormal de communication qui caractérise les familles de schizophrènes. (Dictionnaire fondamental de la psychologie, Paris, Larousse, 1977, p. 386.) 16. Ces remarques ont été faites plusieurs fois, à peu près dans les mêmes termes, par les personnes sollicitées de produire une définition.

17. Télérama 3-10 oct. 1998, nº 2542.

18. Pour une analyse détaillée, voir $P$. Cheminée, op. cit.

19. Mortureux M. Fr., op. cit., p. 48.

20. On reprend ici l'opposition établie par G. Kleiber entre dénomination, "association référentielle durable", codée, entre un signe et un objet, fait de langue, et désignation, association référentielle accidentelle, instable, fait de discours, qui «ne présuppose aucun codage antérieur ». (Voir Kleiber G., "Dénomination et relations dénominatives », in La dénomination, Langages $\mathrm{n}^{\circ}$ 76, Paris, Larousse, 1984, pp. 77-94.)

21. Grand Robert et Dictionnaire historique de la Langue Française.

22. Proust Marcel, Le Temps retrouvé, Paris, éd. Gallimard, coll. «Bibliothèque de la Pléäde », t. III, p. 765.

23. Nouveau Larousse illustré, 7 vol., publié sous la direction de Cl. AUGÉ, éd. Larousse, Paris, 1897-1904.

24. Benveniste Émile, Problèmes de linguistique générale, Paris, éd. Gallimard, coll. « Tel », 1974, t. II, p. 174.

25. Milner J. Cl., «Réflexions sur la référence », in Lexique et grammaire, Langue française $\mathrm{n}^{\circ} 30$, Paris, Larousse, 1976, pp. 63-73.

26. V. l'article de B. Bosredon «Prédicativité et lexicalisation", in Studia Romanica Posnaniensia, vol. 16, U.A.M., Poznan, 1991, pp. 86-100.

27. Pour beaucoup de Français, le "camp de concentration" est spécifiquement nazi et confondu, de surcroît, avec le "camp d'extermination ». Ce qui n'empêche pas, de-ci, de-là, de trouver des attestations 
d'emplois renvoyant à des réalités différentes (Simone Bonafous, Des mots en liberté, in «Mélanges Maurice Tournier », t. I, E.N.S. éd. Fontenay / St-Cloud, 1998, p. 40).

28. Branca-Rosoff Sonia, "Le mot comme notion hétérogène " in Le mot: analyse du discours et sciences sociales, Langues et langage $\mathrm{n}^{\circ}$ 7, Aix-en-Provence, P.U.P. 1998, pp. 7-39. Dans cet article, l'auteur montre, à propos du syntagme guerre sainte, "dénomination figée, élaborée dans des circonstances précises», comment «le recul de la fonction référentielle, au profit d'une définition motivante générale » n'a été possible qu'à partir du changement d'univers discursif, et notamment à partir du « recul de l'espace chrétien ».

29. Duby Georges, Atlas historique, Paris, Librairie Académique Perrin, 1987, p. 481.

30. Duby Georges, Histoire de la France, Paris, éd. Larousse, 1991, vol. III, p. 290.

31. Kleiber G., op. cit. pp. 82-83.

32. Selon le mot employé par Francine Mazière dans son article "Le mot au risque du dictionnaire. Sommes-nous tous des étrangers de la langue maternelle? ", in $D u$ dire et du discours, Hommage à Denise Maldidier, Linx , CRL, Université de Paris X Nanterre, nº 8 spécial, 1996, pp. 13-23.

33. Id., p. 15.

34. id., p. 20.

35. Cf. bibliographie.

36. Qui, tel le langage, ne dit, ni ne cache rien, mais [...] signifie! (PLG II, p. 229).

37. Dubois Cl. et J., op. cit., p. 63.

38. La définition, qui est une longue description des référents possibles, permet d'intégrer toute forme de camp de concentration: «Camp dans lequel sont rassemblés, sous surveillance militaire ou policière, soit des populations civiles de nationalité ennemie, soit des minorités sociales, ethniques ou religieuses, soit des prisonniers ou des détenus politiques » (PL 1991).

39. « où furent affamés, suppliciés et exterminés certains groupes ethniques (Juifs), politiques et sociaux».

40. À ce sujet, signalons le traitement de Purification ethnique dans le PL 94 : ce composé de type N + adj., a trois noms tête en concurrence, alors que la détermination adjectivale s'est tout de suite stabilisée. Voici la définition du PL: «Purification ethnique: élimination, par une population dominante, des autres groupes ethniques vivant sur le même territoire, par l'exercice d'une violence physique ou psychologique. (On dit aussi épuration ethnique et nettoyage ethnique) ». Très curieusement, dans cet enregistrement un peu hâtif, où le syntagme constitue une acception numérotée (2), le dictionnaire en fait une pure dénomination, au point d'effacer les données historiques immédiates, ce qui n'est pas encore fait pour les syntagmes de la Seconde Guerre mondiale. Le PR, en 1992, parlait d'un "Motif donné par le gouvernement serbe dans le conflit... », ce qui en fait un "nom donné à", par un énonciateur précis. L'édition du PL 98 a fait machine arrière, en rentrant le syntagme sous ethnique, en locution à l'intérieur d'un sens : «

Purification, épuration ethniques: noms donnés à l'entreprise d'appropriation exclusive... ». Le statut de la locution a donc changé, puisqu'elle reçoit une définition métalinguistique. Cependant, l'énonciateur reste effacé.

41. Rey Alain, Le Lexique : images et modèles, Paris, Armand Colin, 1977, p. 24.

42. PL 1998 : 3. Espace clos et gardé, aménagé afin de regrouper des personnes en grand nombre et d'en disposer au mépris des droits fondamentaux et à l'abri des regards. (De *travail, de *concentration ou $d^{\prime *}$ extermination, les camps auront été une pièce maîtresse des systèmes totalitaires $d u \mathrm{XX}^{e}$ siècle.) 


\section{RÉSUMÉS}

Pour avoir le statut d'unité lexicale, une séquence doit pouvoir à la fois signifier et référer. Ce sont les deux valeurs du mot pour la linguistique. Pour être reconnue comme une dénomination, l'unité doit avoir un signifiant, un signifié et un référent stables. Le dictionnaire, en intégrant les mots, transforme les unités discursives en unités de lexique, leur garantissant ainsi un statut, sinon lexical, du moins lexicographique. L'étude d'un nom composé morphologiquement instable et de syntagmes que leur mode de référenciation apparente au nom propre montre que statut lexical et statut lexicographique ne recouvrent pas la même réalité.

To be considered as a lexical item, a sequence must be able both to signify and to refer, which are, in linguistics, the two values attached to words. To be recognized as a denomination, each item must have a stable signifier, a stable signified and a stable referent. Dictionaries integrate words by transforming discursive units into lexical items, thereby affording them a status which is, if not lexical, at least lexicographic. The examination of composed words that are morphologically instable, and of syntagmata whose mode of reference is close to that of proper nouns, offers proof that the terms lexical item and lexicographic entry do not cover the same things.

\section{AUTEUR}

\section{PASCALE CHEMINÉE}

11, rue Houdon

75018 Paris

Larousse 